BMJ Open

Diabetes

Research

\& Care

\section{Association of the IgG $N$-glycome with the course of kidney function in type 2 diabetes}

To cite: Singh SS, Heijmans R, Meulen CKE, et al. Association of the lgG $\mathrm{N}$-glycome with the course of kidney function in type 2 diabetes. BMJ Open Diab Res Care 2020;8:e001026. doi:10.1136/ bmjdrc-2019-001026

- Additional material is published online only. To view please visit the journal online (http://dx.doi.org/10.1136/ bmjdrc-2019-001026).

Received 4 November 2019 Revised 5 March 2020 Accepted 6 April 2020
Check for updates

(C) Author(s) (or their employer(s)) 2020. Re-use permitted under CC BY. Published by BMJ.

${ }^{1}$ Internal Medicine, Erasmus MC, Rotterdam, Zuid-Holland, Netherlands

${ }^{2}$ Internal Medicine, Maxima Medical Centre, Eindhoven, Noord-Brabant, Netherlands ${ }^{3}$ Faculty of Pharmacy and Biochemistry, University of Zagreb, Zagreb, Croatia

Correspondence to Dr Mandy van Hoek; m.vanhoek@erasmusmc.n

\section{ABSTRACT}

Introduction Inflammatory processes are thought to be involved in kidney function decline in individuals with type 2 diabetes. Glycosylation of immunoglobulin $\mathrm{G}$ $(\mathrm{lgG})$ is an important post-translation process affecting the inflammatory potential of IgG. We investigated the prospective relationship between Ig $\mathrm{G}$-glycosylation patterns and kidney function in type 2 diabetes. Research design and methods In the DiaGene study, an all-lines-of-care case-control study ( $n=1886)$ with mean prospective follow-up of 7.0 years, the association between 58 IgG $\mathrm{N}$-glycan profiles and estimated glomerular filtration rate (eGFR) and albumin-to-creatinine ratio (ACR) per year and during total follow-up was analyzed. Models were adjusted for clinical variables and multiple comparisons.

Results Eleven traits were significantly associated with eGFR change per year. Bisecting GIcNAc in fucosylated and fucosylated disialylated structures and monosialylation of fucosylated digalactosylated structures were associated with a faster decrease of eGFR. Fucosylation of neutral and monogalactosylated structures was associated with less eGFR decline per year. No significant associations between IgG glycans and ACR were found.

Conclusions In type 2 diabetes, we found IgG $\mathrm{N}$ glycosylation patterns associated with a faster decline of kidney function, reflecting a pro-inflammatory state of IgG. eGFR, but not ACR, was associated with IgG glycans, which suggests these associations may represent renal macroangiopathy rather than microvascular disease.

\section{INTRODUCTION}

Chronic kidney disease (CKD) is one of the most common complications in type 2 diabetes mellitus, despite extensive preventive efforts. Apart from known risk factors, a large residual risk remains for developing CKD, ${ }^{1}{ }^{2}$ which may be partly explained by processes such as inflammation. Biomarkers that provide information, in addition to known risk factors, can aid in better prediction and tailored treatment to prevent and delay kidney function decline. ${ }^{34}$

A growing body of literature recognizes the association between type 2 diabetes and the $N$-linked glycosylation of proteins. ${ }^{5} \mathrm{~N}$-Linked glycosylation is a co-translational and

\section{Significance of this study}

What is already known about this subject?

- Inflammatory processes play a role in chronic kidney disease in type 2 diabetes.

- The variation in glycan sugar residues attached to the conserved glycosylation sites of the immunoglobulin G (IgG) Fc part influences IgG effector function, modulating the immune response from either pro-inflammatory to an anti-inflammatory response or vice versa.

- The link between IgG glycosylation and renal function in type 2 diabetes has never been investigated.

What are the new findings?

- We found pro-inflammatory IgG $\mathrm{N}$-glycosylation patterns associated with a faster decline of kidney function estimated glomerular filtration rate, but not albumin-to-creatinine ratio, possibly representing renal macroangiopathy.

How might these results change the focus of research or clinical practice?

> Our findings suggest the involvement of the immune system in the pathophysiology of diabetic nephropathy in type 2 diabetes, representing a novel target for future biomarker and therapeutics developments.

post-translational modification of proteins, influencing their function. ${ }^{67} \mathrm{~N}$-Glycans affect the stability, activity and targeting of proteins, as well as cell-cell and host-pathogen interaction. $^{6} 7$ These complex oligosaccharides are assembled by the coordinated action of a range of glycosyltransferases and glycosidase enzymes, and are attached to the nitrogen $(\mathrm{N})$ atom of asparagine side chains of proteins within a specific sequon. ${ }^{6} \mathrm{~N}$-Glycosylation patterns of the IgG $\mathrm{N}$-glycome and total plasma $\mathrm{N}$-glycome have been associated with estimated glomerular filtration rate (eGFR) in non-diabetic individuals and those with type 1 diabetes. ${ }^{8}$ Furthermore, a cross-sectional study showed that characteristic patterns of the total plasma $\mathrm{N}$-glycome are associated with renal function in type 2 
diabetes. ${ }^{10}$ However, the link between the immunoglobulin G (IgG) $N$-glycome and renal function in type 2 diabetes patients has never been investigated. Immunoglobulin $\mathrm{G}$ ( $\operatorname{IgG})$ is the most abundant antibody in the human body, involved in infectious and inflammatory processes through several mechanisms: antigen neutralization, promotion of phagocytosis, microbial killing via opsonization and macrophage activation, complement activation and induction of ADCC (antibody-dependent cellular cytotoxicity). ${ }^{11}$ IgG consist of a fragment antigen binding (Fab) domain and fragment crystallizable (Fc) domain, which interacts with Fc gamma receptors (FcyR). Single biantennary glycans are attached to each heavy chain on the Fc part asparagine-297 (Asn297). They are essential for binding to the FcyRs. The receptor interaction is lost if no glycans are attached. The variation in glycan sugar residues attached influences IgG effector function, modulating the immune response from either pro-inflammatory to an anti-inflammatory response or vice versa. The variations consist of the addition of bisecting $N$-acetylglucosamine (GlcNac), fucose to core, as well as galactose and sialic acid to the arms of the biantennary glycan. Bisecting GlcNac and afucosylated $\mathrm{N}$-glycans have a pro-inflammatory effect, while the addition of galactose and sialic acid has an anti-inflammatory effect on IgG (figure 1). ${ }^{12} \operatorname{IgG}$ glycosylation patterns are highly variable between individuals, but show good temporal stability in a single healthy individual. ${ }^{13}$ Yet, IgG patterns are known to change in a single individual because of alterations in a person's health status. ${ }^{13-15} \mathrm{IgG}$ glycosylation contains a genetic, heritable component, as well an environmental component. Because of these features, glycosylation is considered an interface between genetic background and environment. ${ }^{1416121718}$ Because type 2 diabetes and chronic kidney disease are multifactorial diseases displaying features of chronic inflammation, IgG $\mathrm{N}$-glycosylation is promising as a biomarker, but also from a pathophysiological perspective. ${ }^{19}$

We hypothesized that specific IgG-glycan profiles prospectively associate with kidney function in type 2 diabetes, and could be a potential biomarker in the future We, therefore, investigated the prospective relationship between IgG $N$-glycosylation and the course of kidney function during follow-up in type 2 diabetes individuals.
Legend: anti-inflammatory

core-fucosylation

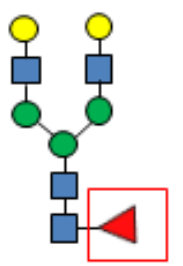

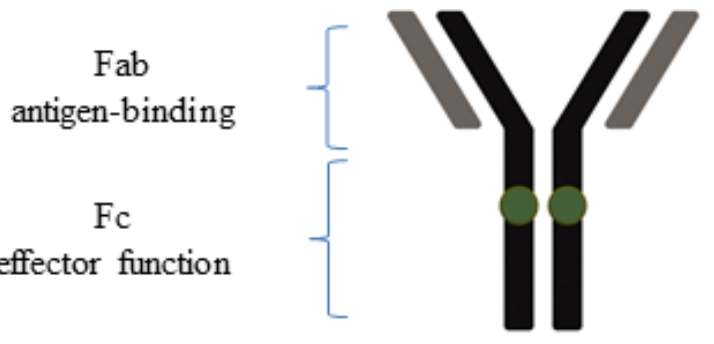

galactosylation

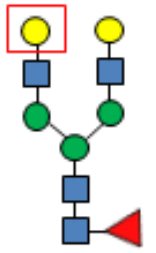

pro-inflammatory

without core fucose

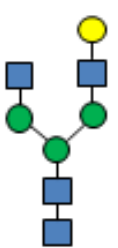

bisecting GLcNAc

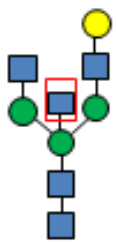

agalactosylation

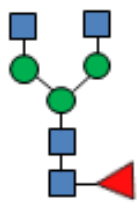

Figure 1 Functional implications and inflammatory associations of lgG glycosylation. Dark-green spots on lgG molecule are conserved glycosylation sites Asn297 to which biantennary glycans are attached. 


\section{RESEARCH DESIGN AND METHODS}

\section{Study design}

Data were derived from the DiaGene study, and the study characteristics have previously been described in more detail. ${ }^{20}$ Briefly, the DiaGene study is a large multicenter prospective cohort study with 1886 patients with type 2 diabetes with prospective follow-up on kidney function (mean follow-up time $=7.0$ years). Data were collected from two hospitals in and around the city of Eindhoven, the Netherlands. In addition, the local primary care diagnostic center participated in the study. All participants provided informed written consent.

\section{Definitions}

Type 2 diabetes was defined as fasting plasma glucose $\geq 7.0 \mathrm{mmol} / \mathrm{L}$ and/or a non-fasting plasma glucose level $\geq 11.1 \mathrm{mmol} / \mathrm{L}$ measured at least at two separate time points, treatment with oral glucose-lowering medication or insulin, and/or the diagnosis of type 2 diabetes as registered by a medical specialist. Microalbuminuria was defined as urinary albumin-to-creatinine ratio (ACR) $\geq 2.5$ for men or $\geq 3.5$ for women. Macroalbuminuria was defined as ACR $\geq 12.5$ for men or $\geq 17.5$ for women. Around the moment of inclusion, laboratory data were obtained, which that is, contained glycated hemoglobin (HbAlc), total cholesterol, high-density lipoprotein (HDL) cholesterol and urinary ACR. The eGFR was calculated with the Modification of Diet in Renal Disease formula and mean arterial pressure (MAP) was defined as (systolic blood pressure+2×diastolic blood pressure)/3. Non-HDL was calculated by subtracting HDL from total cholesterol. To calculate eGFR and ACR percentage change during total follow-up, the value at baseline and the last known value during follow-up were used. The eGFR and ACR percentage change per year were determined by dividing the percentage difference during total follow-up by total follow-up time in years.

\section{IgG $\mathrm{N}$-glycome analysis}

Plasma for IgG $\mathrm{N}$-glycosylation analysis was available in 1837 cases, and 22 samples failed quality control, resulting in 1815 cases. Isolation, release and labeling of IgG glycans has been described in detail previously. ${ }^{5} 14$ In total, 24 IgG glycan peaks were measured by Waters Acquity UPLC instrument. All chromatograms were separated into 24 peaks and the amount of glycans in each peak was expressed as percentage of total integrated area. From these direct traits, an additional 34 derived IgG glycan traits were calculated based on their structural similarities. As a result, characteristics of the 24 direct glycan peaks are reflected in the derived traits. A detailed description can be found in online supplementary table S1. The distribution of the IgG glycan peaks was analyzed by visual inspection of QQ plots and showed no major deviations from normality. IgG glycan expressions were globally normalized and $\log$ transformed, and all measurements were adjusted for batch effects by applying ComBat (R-package sva). Before statistical analyses were performed, all IgG glycan traits were centered and scaled to have mean 0 and SD 1.

\section{Glycan nomenclature}

A glycan structure formula is described as follows: $\mathrm{FA}_{\mathrm{x}} \mathrm{B}-$ $\mathrm{G}_{\mathrm{x}} \mathrm{S}_{\mathrm{x}}$. The abbreviations stand for $\mathrm{F}=$ fucose, $\mathrm{A}=$ antennae, $\mathrm{B}=$ bisection, $\mathrm{G}=$ galactose and $\mathrm{S}=$ sialic acid, and $\mathrm{x}$ indicates the number of the particular feature. When, for example, $\mathrm{F}$ or $\mathrm{S}$ is not present in the structure formula, the glycan has no fucose or sialic acids attached. For some glycans, for example, GP9 with structure formula FA2[3]G1, [3] means the antennae (A2) are bound on the third carbon bond of mannose of the glycan core.

\section{Statistical analysis}

Mean and SD of cohort characteristics were determined. Linear regression models were used to investigate associations between the 58 IgG glycan patterns and eGFR and ACR. Four dependent variables were constructed: percentage change of eGFR during total follow-up, percentage change ACR during total follow-up, eGFR percentage change per year and ACR percentage change per year. The basic model for each dependent variable included age and sex and their interaction. Different full models were constructed, for adjusting for confounders. These full models always contained smoking, MAP, body mass index (BMI), HbAlc, duration of type 2 diabetes, non-HDL and HDL. In addition, in the full models of dependent variables eGFR and ACR percentage change during total follow-up, we adjusted for duration of follow-up. Furthermore, we additionally adjusted the full models of the ACR analyses for ACE-inhibitor use. We had $80 \%$ power to detect Cohen's effect size $f^{2}: 0.009$ based on 1815 cases and 11 predictors. ${ }^{21}$ Correction for multiple comparisons was performed by the BenjaminiHochberg method. ${ }^{22}$ Statistical analyses were carried out using IBM SPSS Statistics V.25.0.

\section{RESULTS}

\section{Cohort characteristics}

Characteristics of the study population are shown in table 1. All participants were aged between 27 and 94 with a mean of 65.2 years; $53.6 \%$ were male, mean BMI was $30.5 \mathrm{~kg} / \mathrm{m}^{2}$, mean $\mathrm{HbAlc}$ was $7.0 \%$ and mean duration of type 2 diabetes at inclusion was 10.1 years. On average, the eGFR and ACR percentage change per year were $-2.3 \%$ and $162.2 \%$, respectively. Microalbuminuria was present in $2.8 \%$ of all patients at baseline and in $5.5 \%$ at end of follow-up. Macroalbuminuria was present in $0.2 \%$ of the patients at both instances.

\section{IgG glycan associations with eGFR percentage change per year}

In table 2, significant associations between IgG $\mathrm{N}$-glycans and eGFR percentage change per year are shown for both models. Results for all 58 investigated IgG glycan peaks and derived traits are shown in online supplementary table S2. 
Table 1 Characteristics of the study population

\begin{tabular}{|c|c|}
\hline Characteristic & Cases $(n=1886)$ \\
\hline Age (years) & $65.2( \pm 10.6)$ \\
\hline Sex (\% male) & 53.6 \\
\hline $\operatorname{BMI}\left(\mathrm{kg} / \mathrm{m}^{2}\right)$ & $30.5( \pm 5.4)$ \\
\hline HDL cholesterol (mmol/L) & $1.17( \pm 0.32)$ \\
\hline Non-HDL cholesterol (mmol/L) & $3.12( \pm 0.90)$ \\
\hline Never smoked (\%) & 23.3 \\
\hline Former smoker (\%) & 50.7 \\
\hline Current smoker (\%) & 16.2 \\
\hline MAP $(\mathrm{mm} \mathrm{Hg})$ & $98.9( \pm 10.8)$ \\
\hline HbA1c (\%) & $7.0( \pm 1.1)$ \\
\hline $\mathrm{HbA1c}(\mathrm{mmol} / \mathrm{L})$ & $53.31(11.58)$ \\
\hline $\begin{array}{l}\text { Duration type } 2 \text { diabetes at inclusion } \\
\text { (years) }\end{array}$ & $10.1( \pm 8.4)$ \\
\hline Duration of follow-up (years) & $6.9( \pm 2.1)$ \\
\hline eGFR change during total follow-up (\%) & $-7.7( \pm 24.5)$ \\
\hline eGFR change per year (\%) & $-2.3( \pm 24.9)$ \\
\hline ACR change during total follow-up (\%) & $800.5( \pm 3626.8)$ \\
\hline ACR change per year (\%) & $162.2( \pm 678.5)$ \\
\hline Normoalbuminuria (ACR <30 mg/g) (\%) & 90.5 \\
\hline Microalbuminuria (ACR 30-300 mg/g) (\%) & 2.8 \\
\hline Macroalbuminuria (ACR >300 mg/g) (\%) & 0.2 \\
\hline Normoalbuminuria at follow-up (\%) & 79.2 \\
\hline Microalbuminuria at follow-up (\%) & 5.5 \\
\hline Macroalbuminuria at follow-up (\%) & 0.2 \\
\hline
\end{tabular}

Unless stated otherwise, mean $( \pm S D)$ are given.

ACR, albumin-to-creatinine ratio; BMI, body mass index; eGFR, estimated glomerular filtration rate; HDL, high-density lipoprotein; MAP, mean arterial pressure.

In the full model, $1 \mathrm{IgG} N$-glycan peak and 10 derived traits were significantly associated with eGFR percentage change per year. Agalactosylated fucosylated biantennary glycans with bisecting GlcNAc (GP6; FA2B) were associated with a faster decrease of eGFR. In addition, bisecting GlcNAc in fucosylated disialylated structures (FBS2/ FS2 and FBS2/(FS2+FBS2)), fucosylated structures with bisecting GlcNAc (FBn, FBG1n/G1n, FBG2n/G2n, FBn/ Fn and FBn/Fntotal) and the percentage of monosialylation of all fucosylated digalactosylated structures without bisecting GlcNAc (FG2S1/(FG2+FG2S1+FG2S2)) were also associated with a faster decrease of eGFR.

Traits associated with less eGFR decline per year were fucosylated structures without bisecting GlcNAc in neutral (Fn) and monogalactosylated structures (FG1n/ G1n).

\section{IgG glycan associations with eGFR percentage change during total follow-up}

Associations between all 58 investigated IgG glycan patterns and the eGFR percentage change during total follow-up are shown in online supplementary table S3.
In the basic model, five IgG $N$-glycan peaks and three derived traits were significantly associated with eGFR percentage change during total follow-up. However, after the adjustment for clinical risk factors, no associations remained significant. In the basic model, patterns associated with a larger decrease in eGFR over time were fucosylated biantennary glycans with (GP6: FA2B) and without bisecting GlcNAc (GP4: FA2) and agalactosylated structures (G0n). Monogalactosylated (GP8: A2BG1, FA2[6]G1; GP9: FA2[3]G1), digalactosylated (GP14: FA2G2), core fucosylated biantennary glycans without bisecting GlcNAc and monogalactosylated (G1n) and digalactosylated structures (G2n) were associated with a smaller decrease in eGFR over time.

\section{IgG glycan associations with ACR}

In both models, we did not find any significant association between IgG glycan patterns and both ACR percentage change and ACR percentage change per year. Associations between all 58 investigated IgG glycan patterns and the ACR percentage change during total follow-up and per year are shown in online supplementary table $\mathrm{S} 4$ and S5, respectively.

\section{DISCUSSION}

In the present study, we describe the prospective association of IgG $N$-glycome with the prospective course of kidney function in type 2 diabetes. After adjustment for confounders, monosialylation, bisecting GlcNAc and fucosylation with bisecting GlcNAc were associated with a faster decrease of eGFR per year. Bisecting GlcNAc and fucosylated bisected IgG reflect biological aging as well as a pro-inflammatory state of IgG. In contrast, fucosylation without bisecting GlcNAc was associated with less eGFR decline per year. These non-bisected fucosylated IgG can implicate an anti-inflammatory IgG effector function. No significant associations were found between IgG glycan patterns and ACR.

Although the pathogenesis of nephropathy in diabetes is only partly understood, it is known that hyperglycemia leads to activation of inflammatory pathways, which attributes to the risk of vascular complications due to tissue injury. ${ }^{23}$ More specifically, in diabetic nephropathy, high blood glucose levels cause renal cells to release humoral mediators, pro-inflammatory cytokines and growth factors. ${ }^{25}{ }^{26}$ Subsequently, GFR deteriorates due to changes in kidney structure and function, such as thickening of the glomerular basement membrane and glomerulosclerosis. ${ }^{25-27}$ Thus, inflammation plays a major role in the development of diabetic nephropathy. Changes in IgG glycan composition can modify the effector function of IgG, hereby contributing to inflammation. ${ }^{28}$ The role of IgG glycans in diabetic nephropathy is supported by an in vivo study, in which Fc $\gamma$ receptor-deficient mice developed less renal hypertrophy, inflammation and fibrosis. ${ }^{29}$ IgG $\mathrm{N}$-glycans change the Fcy receptor affinity, hereby 
Table 2 Statistically significant associations of IgG glycan traits with eGFR change per year

\begin{tabular}{|c|c|c|c|c|}
\hline Glycan traits & Basic model & & Full model & \\
\hline & $\beta$ & $P$ value & $\beta$ & $P$ value \\
\hline GP4 (FA2) & -1.13 & $6.17 \mathrm{E}-03$ & -0.13 & NS \\
\hline GP6 (FA2B) & -1.38 & 7.76E-04 & -0.76 & $6.18 \mathrm{E}-03$ \\
\hline GP8 (A2BG1; FA2[6]G1) & 1.18 & $2.28 \mathrm{E}-03$ & 0.61 & NS \\
\hline GP9 (FA2[3]G1) & 1.05 & $5.65 \mathrm{E}-03$ & 0.44 & NS \\
\hline GP14 (FA2G2) & 1.12 & $1.01 \mathrm{E}-02$ & 0.49 & NS \\
\hline \multicolumn{5}{|l|}{ Derived traits } \\
\hline $\mathrm{FG} 2 \mathrm{~S} 1 /(\mathrm{FG} 2+\mathrm{FG} 2 \mathrm{~S} 1+\mathrm{FG} 2 \mathrm{~S} 2)$ & -0.27 & NS & -0.74 & $2.66 \mathrm{E}-03$ \\
\hline FBStotal/FStotal & -1.16 & $3.94 \mathrm{E}-03$ & -0.31 & NS \\
\hline FBS2/FS2 & -1.38 & 4.16E-04 & -0.78 & $3.20 \mathrm{E}-03$ \\
\hline $\mathrm{FBS} 2 /(\mathrm{FS} 2+\mathrm{FBS} 2)$ & -1.21 & $2.25 \mathrm{E}-03$ & -0.74 & $5.89 \mathrm{E}-03$ \\
\hline GOn & -1.32 & $1.84 \mathrm{E}-03$ & -0.39 & NS \\
\hline G1n & 1.43 & $3.51 \mathrm{E}-04$ & 0.34 & NS \\
\hline Fn & 0.71 & NS & 0.70 & $5.70 \mathrm{E}-03$ \\
\hline $\mathrm{FG} 1 \mathrm{n} / \mathrm{G} 1 \mathrm{n}$ & 0.80 & NS & 0.71 & 4.71E-03 \\
\hline $\mathrm{FG} 2 \mathrm{n} / \mathrm{G} 2 \mathrm{n}$ & 1.08 & $6.09 \mathrm{E}-03$ & 0.51 & NS \\
\hline FBn & -0.73 & NS & -0.72 & 4.73E-03 \\
\hline FBG1n/G1n & -0.77 & NS & -0.70 & $5.24 \mathrm{E}-03$ \\
\hline FBG2n/G2n & -1.27 & $1.42 \mathrm{E}-03$ & -0.75 & 4.11E-03 \\
\hline $\mathrm{FBn} / \mathrm{Fn}$ & -0.77 & NS & -0.78 & $2.20 \mathrm{E}-03$ \\
\hline FBn/Fntotal & -0.74 & NS & -0.74 & 3.89E-03 \\
\hline
\end{tabular}

Basic model: age, sex and their interaction.

Full model: smoking, mean arterial pressure, body mass index, HbA1c, duration of type 2 diabetes, non-HDL, HDL.

A $p$ value was considered statistically significant for the basic model when $p<1.01 E-02$ and for the full model when $p<6.18 E-03$. Regression coefficient (beta) and $p$ value per association, with FDR $\alpha$ used as cut-off for significance.

B, bisection; eGFR, estimated glomerular filtration rate; F, fucose; G, galactose; HDL, high-density lipoprotein; N, neutral; NS, non-significant; $\mathrm{S}$, sialylation.

regulating inflammatory processes such as antibodydependent cellular phagocytosis and ADCC. IgG glycan modulation has also been shown to attenuate the development of ANCA-mediated glomerulonephritis. ${ }^{30}$ IgG $N$-glycosylation patterns have already been associated with kidney function in non-diabetic individuals and type 1 diabetes. ${ }^{89}$ Key properties of IgG glycosylation are galactosylation, sialylation, fucosylation and bisecting GlcNAc. We will discuss our findings and associated literature accordingly.

Galactosylation of IgG $N$-glycans is essential for the initiation of the anti-inflammatory signaling cascade through the inhibitory receptor FcyRIIB. ${ }^{31}$ In addition, the proinflammatory activity of the complement component C5a can be inhibited by highly galactosylated immune complexes. $^{28} 3233$ Therefore, galactosylation reflects an anti-inflammatory state of IgG. A lack of galactosylation is known to activate the complement system via the lectin pathway by binding to mannose-binding lectin and via the alternative pathway, ${ }^{28234-36}$ which induces inflammation. In our study, agalactosylated structures were associated with a faster decline of kidney function, whereas monogalactosylated structures and GP14 (FA2G2) were associated with less kidney function decline per year in the basic model. When adjusting for clinical covariates, such as HbA1c, these associations did not remain significant. However, in the extensive model, GP6 (FA2B), of which the absence of galactose is an important feature, was significantly associated with a faster decline of kidney function independent of clinical covariates. These findings are in line with other studies, as agalactosylated $\operatorname{IgG}$ glycan structures were also associated with a more rapid eGFR decline in type 1 diabetes and a higher risk of CKD in the non-diabetic population. ${ }^{89}$

In previous studies, the sialylation of IgG glycans decreased the affinity of Fc $\gamma$ receptors, leading to antiinflammatory activity ${ }^{187}$ : the addition of sialic acid alters IgG from a pro-inflammatory to an anti-inflammatory state. In our study, GP6 (FA2B), characterized by the absence of sialic acid, was associated with faster decrease in kidney function. In line with our finding, the percentage of sialylated structures in IgG glycans was also decreased in non-diabetic patients with CKD. ${ }^{9}$ In our study, a faster decrease in kidney function was also associated with monosialylation of fucosylated digalactosylated structures without bisecting GlcNAc, which 
we cannot completely explain. It could be a result of a relative increase, as a consequence of a (non-significant) reduction in disialylated structures of the same type. This is supported by the overall negative direction of association of the percentage of sialylation in these structures.

High abundance of IgG core fucose serves as a "safety switch", as it decreases the affinity for the Fc $\gamma$ RIIIA and Fc $\gamma$ RIIIB receptors. ${ }^{38-40}$ As a result, core fucose prevents ADCC. ${ }^{38-40}$ However, the presence of bisecting GlcNac in fucosylated structures has the opposite effect, as it increases the affinity for the receptors mentioned above. ${ }^{414}$ The combination of fucose with bisecting GlcNac is associated with a pro-inflammatory state of IgG. In our study, fucosylated structures with bisecting GlcNAc were associated with a faster decrease of kidney function after adjustment for clinical variables. This was also reflected in the association with GP6 (FA2B), which entails the same feature. Moreover, fucosylated structures without bisecting GlcNAc were associated with less annual kidney function decline. Similar results were found in a non-diabetic population, as sialylated core fucosylated glycans with bisecting GlcNac were associated with lower eGFR. ${ }^{8}$ Interestingly, monosialylation with presence of bisecting GlcNac was associated with lower eGFR in the non-diabetic population, ${ }^{8}$ whereas in our study, disialylation in combination with bisecting GlcNAc was associated with a faster decrease of eGFR.

In our study, we did not find an association of $\operatorname{IgG}$ glycosylation with prospective changes in albuminuria. Increase of ACR is one of the first clinical signs of diabetic nephropathy. However, studies in both types of diabetes have also shown that higher albuminuria levels do not necessarily result into renal function decline. ${ }^{43-45}$ A portion of diabetic patients indeed presents with normoalbuminuria in combination with a decline in renal function. ${ }^{46}{ }^{47}$ It has been shown that these patients tend to have a higher age and a more advanced CKD with lower eGFR and lower hemoglobin. ${ }^{48}$ This suggests that, for these patients, the kidney function decline may be related to macrovascular rather than to microvascular disease, as may also be the case in our population of older patients with type 2 diabetes. As another study ${ }^{45}$ has shown that intrarenal vascular disease is dependent of eGFR, but independent of ACR, the associations between IgG glycans and renal function found in our study may indicate renal macroangiopathy rather than microvascular glomerulopathy. Associations between IgG glycans and ACR were described in type 1 diabetes. ${ }^{9}$ The large portion of both microalbuminuria and macroalbuminuria at baseline in this type 1 diabetes population (13.45\% and $10.27 \%$ vs $2.8 \%$ and $0.2 \%$ in our study, respectively) may explain this difference.

Strengths of our study are the use of a large and unselected cohort of patients with type 2 diabetes from all lines of care with prospective follow-up. A variety of clinical features was available to include in our analysis. Furthermore, this is the first study addressing the IgG $\mathrm{N}$-glycome in relation to kidney disease in type 2 diabetes and prospectively. Although we have performed our study with great care, some limitations remain. First, IgG glycans were measured at baseline. As a result, we have not investigated temporal sequence glycan composition changes, which could have influenced the kidney function during follow-up. However, IgG glycans are known to be fairly stable unless a major physiological change takes place. ${ }^{13}$ Moreover, for biomarker purposes, a marker that predicts well with one measurement, rather than sequential measurements would be preferred. Second, residual confounding due to yet unknown factors affecting both the IgG $N$-glycome and kidney function cannot be excluded. However, by adjusting for many clinical factors, we have reduced this to a minimum. Third, our study has only evaluated the IgG $\mathrm{N}$-glycome, which reflects glycans originating from IgG. Glycans present on other plasma proteins, as can be measured in the total $\mathrm{N}$-glycome, may also associate with the prognosis of kidney function.

To conclude, we found IgG $N$-glycosylation patterns, reflecting a pro-inflammatory state of IgG at baseline, associated prospectively with a faster decline of kidney function in patients with type 2 diabetes. eGFR, but not ACR, was associated with IgG glycans, which suggests these associations may represent renal macroangiopathy rather than microvascular disease.

Our findings suggest the involvement of the immune system in the pathophysiology of diabetic nephropathy in type 2 diabetes, representing a novel target for future biomarker and therapeutics development. Mendelian randomization studies and in-depth in vitro studies could provide more insight into the causality of the discovered relationships as well as the pathophysiological mechanisms.

Contributors SSS maintained the DiaGene study database, analyzed the data, wrote and reviewed/edited the manuscript. RH, CKEM analyzed and completed the database, wrote and reviewed/edited manuscript. AGL collected and designed the DiaGene Study, reviewed and edited the manuscript. OG, GL reviewed and edited the manuscript. EJGS collected and designed the DiaGene Study, reviewed and edited the manuscript. MvH coordinated the analyses; initiated the research question, collection and design of the DiaGene study; wrote/reviewed and edited the manuscript. All authors have read and approved the final manuscript. MvH is the guarantor of this work and, as such, had full access to all the data in the study and takes responsibility for the integrity of the data and the accuracy of the data analysis.

Funding The authors have not declared a specific grant for this research from any funding agency in the public, commercial or not-for-profit sectors.

Competing interests None declared.

Patient consent for publication Not required.

Ethics approval The study was approved by the Medical Ethical Committees of the Erasmus MC, Catharina Hospital and the Maxima Medical Center (MEC-230).

Provenance and peer review Not commissioned; externally peer reviewed.

Data availability statement The datasets generated during and/or analyzed during the current study are not publicly available. The raw data are subject to "Special Categories of Personal Data (Sensitive Data)" (GDPR, Article 9); therefore, raw data sharing is not in line with the privacy principles. Also, the information provided to the participants in the study states that the individual data are only accessible to the researchers, the ethical review board and (local) authorities. The informed consent given by the participants is therefore not sufficient for open access publication of indirectly identifiable data. Datasets are available from the corresponding author on reasonable request. 
Open access This is an open access article distributed in accordance with the Creative Commons Attribution 4.0 Unported (CC BY 4.0) license, which permits others to copy, redistribute, remix, transform and build upon this work for any purpose, provided the original work is properly cited, a link to the licence is given, and indication of whether changes were made. See: https://creativecommons.org/ licenses/by/4.0/.

ORCID iD

Mandy van Hoek http://orcid.org/0000-0002-2957-5436

\section{REFERENCES}

1 Gaede P, Vedel P, Larsen N, et al. Multifactorial intervention and cardiovascular disease in patients with type 2 diabetes. $N$ Engl $J$ Med 2003;348:383-93.

2 Gaede $\mathrm{P}$, Lund-Andersen $\mathrm{H}$, Parving $\mathrm{H}-\mathrm{H}$, et al. Effect of a multifactorial intervention on mortality in type 2 diabetes. $N$ Engl $J$ Med 2008;358:580-91.

3 International Diabetes Federation. IDF diabetes atlas, 8th edition, key messages, 2017. Available: http://www.diabetesatlas.org/keymessages.html

4 Schernthaner G, Mogensen CE, Schernthaner $\mathrm{G}-\mathrm{H}$. The effects of GLP-1 analogues, DPP-4 inhibitors and SGLT2 inhibitors on the renal system. Diab Vasc Dis Res 2014;11:306-23.

5 Lemmers RFH, Vilaj M, Urda D, et al. IgG glycan patterns are associated with type 2 diabetes in independent European populations. Biochim Biophys Acta Gen Subj 2017;1861:2240-9.

6 Ohtsubo K, Marth JD. Glycosylation in cellular mechanisms of health and disease. Cell 2006;126:855-67.

7 Varki A. Biological roles of glycans. Glycobiology 2017;27:3-49.

8 Barrios C, Zierer J, Gudelj I, et al. Glycosylation profile of IgG in moderate kidney dysfunction. J Am Soc Nephrol 2016;27:933-41.

9 Bermingham ML, Colombo M, McGurnaghan SJ, et al. N-Glycan profile and kidney disease in type 1 diabetes. Diabetes Care 2018;41:79-87.

10 Adua E, Anto EO, Roberts $\mathrm{P}$, et al. The potential of $\mathrm{N}$-glycosylation profiles as biomarkers for monitoring the progression of type II diabetes mellitus towards diabetic kidney disease. J Diabetes Metab Disord 2018;17:233-46.

11 Lu LL, Suscovich TJ, Fortune SM, et al. Beyond binding: antibody effector functions in infectious diseases. Nat Rev Immunol 2018;18:46-61.

12 Lauc G, Pezer M, Rudan I, et al. Mechanisms of disease: the human N-glycome. Biochim Biophys Acta 2016;1860:1574-82.

13 Gornik O, Wagner J, Pucić M, et al. Stability of $\mathrm{N}$-glycan profiles in human plasma. Glycobiology 2009;19:1547-53.

14 Pucić M, Knezević A, Vidic J, et al. High throughput isolation and glycosylation analysis of IgG-variability and heritability of the IgG glycome in three isolated human populations. Mol Cell Proteomics 2011;10:M111.010090-90.

15 Menni C, Keser T, Mangino M, et al. Glycosylation of immunoglobulin G: role of genetic and epigenetic influences. PLoS One 2013;8:e82558.

16 Wahl A, van den Akker E, Klaric L, et al. Genome-wide association study on immunoglobulin G glycosylation patterns. Front Immunol 2018;9:277.

17 Gornik O, Pavić T, Lauc G. Alternative glycosylation modulates function of IgG and other proteins - implications on evolution and disease. Biochim Biophys Acta 2012;1820:1318-26.

18 Kaneko Y, Nimmerjahn F, Ravetch JV. Anti-Inflammatory activity of immunoglobulin $\mathrm{G}$ resulting from Fc sialylation. Science 2006;313:670-3

19 Lontchi-Yimagou E, Sobngwi E, Matsha TE, et al. Diabetes mellitus and inflammation. Curr Diab Rep 2013;13:435-44.

20 van Herpt TTW, Lemmers RFH, van Hoek M, et al. Introduction of the DiaGene study: clinical characteristics, pathophysiology and determinants of vascular complications of type 2 diabetes. Diabetol Metab Syndr 2017;9:47

21 Faul F, Erdfelder E, Buchner A, et al. Statistical power analyses using $\mathrm{G}^{*}$ Power 3.1: tests for correlation and regression analyses. Behav Res Methods 2009;41:1149-60.

22 Benjamini Y, Hochberg Y. Controlling the false discovery rate: a practical and powerful approach to multiple testing. J $R$ Stat Soc Series B 1995;57:289-300.

23 Giacco F, Brownlee M. Oxidative stress and diabetic complications. Circ Res 2010;107:1058-70.
24 Brownlee M. Biochemistry and molecular cell biology of diabetic complications. Nature 2001;414:813-20.

25 Schena FP, Gesualdo L. Pathogenetic mechanisms of diabetic nephropathy. J Am Soc Nephrol 2005;16 Suppl 1:S30-3.

26 Moreno JA, Gomez-Guerrero C, Mas S, et al. Targeting inflammation in diabetic nephropathy: a tale of hope. Expert Opin Investig Drugs 2018;27:917-30.

27 Cheng $\mathrm{H}$, Harris RC. Renal endothelial dysfunction in diabetic nephropathy. Cardiovasc Hematol Disord Drug Targets 2014;14:22-33.

28 Gudelj I, Lauc G, Pezer M. Immunoglobulin G glycosylation in aging and diseases. Cell Immunol 2018;333:65-79.

29 Lopez-Parra V, Mallavia B, Lopez-Franco O, et al. Fc $\gamma$ receptor deficiency attenuates diabetic nephropathy. J Am Soc Nephrol 2012;23:1518-27.

30 van Timmeren MM, van der Veen BS, Stegeman CA, et al. IgG glycan hydrolysis attenuates ANCA-mediated glomerulonephritis. $J$ Am Soc Nephrol 2010;21:1103-14.

31 Karsten CM, Pandey MK, Figge J, et al. Anti-inflammatory activity of IgG1 mediated by Fc galactosylation and association of Fc $\gamma R$ IIB and Dectin-1. Nat Med 2012;18:1401-6.

32 Malhotra R, Wormald MR, Rudd PM, et al. Glycosylation changes of IgG associated with rheumatoid arthritis can activate complement via the mannose-binding protein. Nat Med 1995;1:237-43.

33 Mihai S, Nimmerjahn F. The role of Fc receptors and complement in autoimmunity. Autoimmun Rev 2013;12:657-60.

34 Banda NK, Wood AK, Takahashi K, et al. Initiation of the alternative pathway of murine complement by immune complexes is dependent on N-glycans in IgG antibodies. Arthritis Rheum 2008:58:3081-9.

35 Arnold JN, Wormald MR, Sim RB, et al. The impact of glycosylation on the biological function and structure of human immunoglobulins. Annu Rev Immunol 2007;25:21-50.

36 Nimmerjahn F, Anthony RM, Ravetch JV. Agalactosylated IgG antibodies depend on cellular Fc receptors for in vivo activity. Proc Natl Acad Sci U S A 2007;104:8433-7.

37 Anthony RM, Ravetch JV. A novel role for the IgG Fc glycan: the antiinflammatory activity of sialylated IgG FCS. J Clin Immunol 2010;30 Suppl 1:9-14

38 Shields RL, Lai J, Keck R, et al. Lack of fucose on human IgG1 $\mathrm{N}$-linked oligosaccharide improves binding to human Fcgamma RIII and antibody-dependent cellular toxicity. J Biol Chem 2002;277:26733-40.

39 Hmiel LK, Brorson KA, Boyne MT. Post-translational structural modifications of immunoglobulin $\mathrm{G}$ and their effect on biological activity. Anal Bioanal Chem 2015;407:79-94.

40 Shinkawa T, Nakamura K, Yamane N, et al. The absence of fucose but not the presence of galactose or bisecting $\mathrm{N}$-acetylglucosamine of human IgG1 complex-type oligosaccharides shows the critical role of enhancing antibody-dependent cellular cytotoxicity. J Biol Chem 2003:278:3466-73.

41 Davies J, Jiang L, Pan LZ, et al. Expression of GnTIII in a recombinant anti-CD20 $\mathrm{CHO}$ production cell line: expression of antibodies with altered glycoforms leads to an increase in ADCC through higher affinity for Fc gamma RIII. Biotechnol Bioeng 2001;74:288-94.

42 Zou G, Ochiai H, Huang W, et al. Chemoenzymatic synthesis and Fc $\gamma$ receptor binding of homogeneous glycoforms of antibody Fc domain. presence of a bisecting sugar moiety enhances the affinity of Fc to Fcyllla receptor. J Am Chem Soc 2011;133:18975-91.

43 Perkins BA, Ficociello LH, Roshan B, et al. In patients with type 1 diabetes and new-onset microalbuminuria the development of advanced chronic kidney disease may not require progression to proteinuria. Kidney Int 2010;77:57-64.

44 Caramori ML, Fioretto P, Mauer M. Low glomerular filtration rate in normoalbuminuric type 1 diabetic patients: an indicator of more advanced glomerular lesions. Diabetes 2003;52:1036-40.

45 Maclsaac RJ, Panagiotopoulos S, McNeil KJ, et al. Is nonalbuminuric renal insufficiency in type 2 diabetes related to an increase in intrarenal vascular disease? Diabetes Care 2006;29:1560-6.

46 Garg AX, Kiberd BA, Clark WF, et al. Albuminuria and renal insufficiency prevalence guides population screening: results from the NHANES III. Kidney Int 2002;61:2165-75.

47 Kramer HJ, Nguyen QD, Curhan G, et al. Renal insufficiency in the absence of albuminuria and retinopathy among adults with type 2 diabetes mellitus. JAMA 2003;289:3273-7.

48 Laranjinha I, Matias P, Mateus S, et al. Diabetic kidney disease: is there a non-albuminuric phenotype in type 2 diabetic patients? Nefrologia 2016;36:503-9. 